\title{
Fotossíntese, relações hídricas e crescimento de cafeeiros jovens em relação à disponibilidade de fósforo
}

\author{
Leandro da Silva(1), Paulo Eduardo Ribeiro Marchiori(1), Cíntia Pinheiro Maciel(1), \\ Eduardo Caruso Machado( ${ }^{(1)}$ e Rafael Vasconcelos Ribeiro(1)
}

(1)Instituto Agronômico, Centro de Pesquisa e Desenvolvimento em Ecofisiologia e Biofísica, Av. Barão de Itapura, $\mathrm{n}^{\circ}$ 1.481, Botafogo, CEP13012-970 Campinas, SP. E-mail: leandropg08@gmail.com, marchiori.paulo@gmail.com, cintiapinheirom@gmail.com, caruso@iac.sp.gov.br, rafael@iac.sp.gov.br

Resumo - O objetivo deste trabalho foi avaliar de que maneira a alta disponibilidade de fósforo no solo afeta a fotossíntese e o crescimento de mudas de cafeeiro arábica (Coffea arabica). Mudas da cultivar Ouro Verde com aproximadamente quatro meses de idade, cultivadas com boa disponibilidade hídrica, foram submetidas a três tratamentos quanto à disponibilidade de fósforo: quantidade recomendada de $\mathrm{P}$, na literatura (PA); duas vezes a dosagem utilizada em $\mathrm{PA}(\mathrm{P}+)$; e sem adição de $\mathrm{P}$ ao solo (P-). Após 70 dias da aplicação dos tratamentos, foram avaliados: as trocas gasosas, a atividade fotoquímica, o potencial de água da folha, a condutância hidráulica da planta $\left(\mathrm{K}_{\mathrm{L}}\right)$, a partição de matéria seca na planta, os teores de pigmentos e carboidratos, e a composição química das folhas. O tratamento P- influenciou negativamente a fotossíntese, e levou à restrição do crescimento das plantas. As plantas do tratamento $\mathrm{P}+$ apresentaram maior teor foliar de $\mathrm{P}\left(\sim 1,9 \mathrm{~g} \mathrm{~kg}^{-1}\right)$, com incrementos na assimilação de $\mathrm{CO}_{2}$, na eficiência instantânea de carboxilação e na atividade fotoquímica - maior eficiência do fotossistema II e maior transporte aparente de elétrons - em relação às plantas do tratamento PA. Houve aumento em $\mathrm{K}_{\mathrm{L}}$, maior teor de carboidratos foliares e maior teor de clorofila nas plantas que receberam o dobro da dose recomendada de $\mathrm{P}$, as quais apresentaram maior produção de matéria seca em relação às de PA e P-.

Termos para indexação: Coffea arabica, adubação fosfatada, carboidratos, fotoquímica, trocas gasosas.

\section{Photosynthesis, water relations and growth of young coffee plants according to phosphorus availability}

\begin{abstract}
The objective of this work was to evaluate how high soil phosphorus (P) availability affects the photosynthesis and growth of young coffee arabica plants (Coffea arabica). Four months old coffee seedlings of the Ouro Verde cultivar, cultivated in good hydric conditions, were exposed to three $\mathrm{P}$ availability treatments: $\mathrm{P}$ dosage recommended in the literature (RP); two times the recommended dosage $(\mathrm{P}+)$; and without $\mathrm{P}(\mathrm{P}-)$. Seventy days after treatment application, evaluations of leaf gas exchange, photochemical activity, leaf water potential, plant hydraulic conductance $\left(\mathrm{K}_{\mathrm{L}}\right)$, dry matter partitioning, leaf contents of pigments and carbohydrates, and leaf chemical composition were done. The treatment without $P$ caused reduction in photosynthesis, leading to lower plant growth. The plants of the $\mathrm{P}+$ treatment showed the highest leaf $\mathrm{P}$ concentrations $\left(\sim 1.9 \mathrm{~g} \mathrm{~kg}^{-1}\right)$, with increasing leaf $\mathrm{CO}_{2}$ assimilation and instantaneous carboxylation efficiency, and higher photochemical activity - higher effective quantum efficiency of photosystem II and apparent electron transport rate - when compared to plants exposed to RP treatment. Plants exposed to the double P recommended dosage exhibited higher $\mathrm{K}_{\mathrm{L}}$, higher leaf carbohydrate content and higher chlorophyll content, resulting in higher dry matter production as compared to RP and P-.
\end{abstract}

Index terms: Coffea arabica, phosphate fertilization, carbohydrates, photochemistry, gas exchange.

\section{Introdução}

O cafeeiro (Coffea arabica L.) é uma planta sensível a desequilíbrios nutricionais e, por isso, tecnologias que permitam maior eficiência produtiva são desejáveis (Reis Junior \& Martinez, 2002). Em relação à adubação fosfatada, sabe-se que a falta desse nutriente causa distúrbios imediatos no metabolismo e no desenvolvimento das plantas (Lawlor \& Cornic, 2002).
Decréscimos da ciclagem de P entre o citoplasma e o estroma, gerados por redução da absorção de P no solo (Hendrickson et al., 2004; Santos et al., 2006), podem levar a diminuições no consumo e na produção de ATP e NADPH, menor carboxilação/regeneração de RuBP (Shubhra et al., 2004), decréscimo na expressão de genes relacionados à fotossíntese (Lawlor \& Cornic, 2002), fechamento estomático (Flügge et al., 2003) e menor condutância do mesofilo (Lawlor \& Cornic, 2002). 
Portanto, o resultado final seria o comprometimento da atividade fotossintética das plantas, com possível redução de seu crescimento e desenvolvimento.

A disponibilidade adequada de $\mathrm{P}$ proporciona cafeeiros bem desenvolvidos e vigorosos, o que, em última instância, determina boas produções. Observações de campo têm demonstrado que há aumento da produtividade com o uso de adubo fosfatado acima das quantidades recomendadas. Todavia, a maior parte dos estudos publicados sobre a presença de $\mathrm{P}$ se referem aos efeitos da baixa disponibilidade desse elemento no metabolismo vegetal, crescimento e nutrição das plantas (López-Bucio et al., 2002; Hendrickson et al., 2004; Reis Junior \& Martinez, 2002), com pouca ênfase aos possíveis efeitos de altas concentrações desse nutriente no solo. Assim, ampliar o conhecimento sobre a fisiologia de cafeeiros em relação à aplicação excessiva de $\mathrm{P}$ no solo é fundamental para $\mathrm{o}$ melhor entendimento do metabolismo desse nutriente nas plantas.

O objetivo deste trabalho foi avaliar como a alta disponibilidade de fósforo $(\mathrm{P})$ no solo afeta a fotossíntese e o crescimento de mudas de cafeeiro arábica em condição de boa disponibilidade hídrica.

\section{Material e Métodos}

Mudas de cafeeiro arábica, cultivar Ouro Verde, com aproximadamente quatro meses de idade, foram plantadas em potes de plástico de $9 \mathrm{dm}^{3}$ e cultivadas em casa-de-vegetação. No momento do transplantio, o solo aderido às raízes das plantas foi retirado para que a disponibilidade inicial de $\mathrm{P}$ fosse homogênea nas plantas de todos os tratamentos. A fase experimental teve início 15 dias após o transplantio, apenas quando as plantas mostraram sintomas de recuperação vegetativa, revelados pelo lançamento de novo par de folhas. As plantas cresceram livres de patógenos e pragas e sob boa disponibilidade hídrica, com o solo mantido próximo a $80 \%$ da capacidade de campo, com irrigações a cada dois dias.

O solo utilizado tinha a seguinte composição química, segundo métodos propostos por Raij et al. (2001): $\mathrm{pH} \mathrm{CaCl} 2$, 4,2; P, 2,0 mg dm${ }^{-3}$; $\mathrm{S}, 14 \mathrm{mg} \mathrm{dm}^{-3}$; $\mathrm{B}, 0,26 \mathrm{mg} \mathrm{dm}^{-3} ; \mathrm{Cu}, 2,0 \mathrm{mg} \mathrm{dm}^{-3} ; \mathrm{Fe}, 55 \mathrm{mg} \mathrm{dm}^{-3}$; $\mathrm{Mn}, 3,0 \mathrm{mg} \mathrm{dm}^{-3}$; Zn, 0,6 mg dm${ }^{-3}$; Al, 5,0 $\mathrm{mmol}_{\mathrm{c}} \mathrm{dm}^{-3}$; $\mathrm{Ca}, 7,0 \mathrm{mmol}_{\mathrm{c}} \mathrm{dm}^{-3} ; \mathrm{Mg}, 3,2 \mathrm{mmol}_{\mathrm{c}} \mathrm{dm}^{-3} ; \mathrm{K}, 1,1 \mathrm{mmol}_{\mathrm{c}}$ $\mathrm{dm}^{-3}$; SB, 11,0 $\mathrm{mmol}_{\mathrm{c}} \mathrm{dm}^{-3}$; CTC (pH 7,0), 50,7 $\mathrm{mmol}_{\mathrm{c}}$ $\mathrm{dm}^{-3} ; \mathrm{V}, 25 \%$ e $\mathrm{MO}, 25 \mathrm{~g} \mathrm{dm}^{-3}$.
A adubação de plantio correspondeu à aplicação dos nutrientes nas seguintes doses em $\mathrm{mg} \mathrm{kg}^{-1} \mathrm{de}$ solo: N, 60; P, 200 e K, 100, fornecidos na forma de uréia, fosfato monoamônico (MAP) e cloreto de potássio, respectivamente. Também foi adicionado calcário dolomítico com PRNT equivalente a $100 \%$, utilizando-se $1 \mathrm{~g} \mathrm{~kg}^{-1}$ de solo. A adubação pós-plantio foi realizada com $\mathrm{N}$ e $\mathrm{K}$ de forma parcelada $-3 \mathrm{~g}$ de $\mathrm{N}$ e de $\mathrm{K}$ por pote - em intervalos de 30 dias, durante dois meses. Após 30 dias do transplantio, procedeu-se ao fornecimento de micronutrientes via solução nutritiva, com a adição de $0,5 \mathrm{mg} \mathrm{kg}^{-1}$ de $\mathrm{B}\left(\mathrm{H}_{3} \mathrm{BO}_{3}\right), 4,7 \mathrm{mg} \mathrm{kg}^{-1}$ de $\mathrm{Zn}\left(\mathrm{ZnCl}_{2}\right), 5 \mathrm{mg} \mathrm{kg}^{-1}$ de $\mathrm{Mn}\left(\mathrm{MnCl}_{2}\right)$ e $1 \mathrm{mg} \mathrm{kg}^{-1} \mathrm{de}$ $\mathrm{Cu}\left(\mathrm{CuSO}_{4} 5 \mathrm{H}_{2} \mathrm{O}\right)$. Macronutrientes e micronutrientes foram fornecidos seguindo as recomendações para o cultivo de cafeeiros (Fazuoli et al., 1998).

As mudas de cafeeiro foram submetidas a três condições de disponibilidade de P: baixa disponibilidade, em que não se realizou adição de $\mathrm{P}$ ao solo (P-); disponibilidade recomendada, com adição de $286 \mathrm{mg} \mathrm{kg}^{-1}$ de $\mathrm{P}_{2} \mathrm{O}_{5}$ (PA); e alta disponibilidade, com o dobro da dosagem utilizada em PA $(\mathrm{P}+)$. A adubação foi balanceada de tal forma que apenas o fósforo fosse variável em todos os tratamentos, e o fornecimento do $\mathrm{P}$ foi realizado na adubação de plantio, juntamente com os demais macronutrientes. Uma curva de incubação do solo com o MAP foi realizada para determinar a quantidade de fósforo disponível e não adsorvido para as plantas de acordo com a quantidade de adubo utilizada.

As plantas cresceram nas três condições de disponibilidade de fósforo até que os cafeeiros do tratamento P- exibissem sintomas de deficiência nutricional, após 70 dias do transplantio das mudas. Nesse momento, foram avaliadas características fisiológicas e biométricas, além do teor foliar de macronutrientes.

As avaliações da variação diurna das trocas gasosas, da fluorescência da clorofila e do potencial da água na folha foram realizadas em folhas maduras totalmente expandidas. As medidas consideraram a variação natural da radiação fotossinteticamente ativa $(\mathrm{Q}$, máximo de $1.600 \mu \mathrm{mol} \mathrm{m} \mathrm{m}^{-2} \mathrm{~s}^{-1}$ ), temperatura entre 26,4 e $36,2{ }^{\circ} \mathrm{C}$ e e deficit de pressão de vapor entre a folha e o ar com variação entre 1,8 e $3,4 \mathrm{kPa}$. A concentração de $\mathrm{CO}_{2}$ no ar foi controlada e constante, de $380 \mu \mathrm{mol} \mathrm{mol}^{-1}$.

Assimilação de $\mathrm{CO}_{2}\left(\mathrm{P}_{\mathrm{N}}\right)$, condutância estomática $\left(\mathrm{g}_{\mathrm{S}}\right)$, concentração intercelular de $\mathrm{CO}_{2}\left(\mathrm{C}_{\mathrm{I}}\right)$ e transpiração 
(E) foram avaliadas com um analisador de gases por infravermelho modelo Li-6400F (LI-COR, Inc., Lincoln, EUA). Os valores de $\mathrm{P}_{\mathrm{N}}$ e $\mathrm{C}_{\mathrm{I}}$ foram utilizados para o cálculo da eficiência instantânea de carboxilação $\left(\mathrm{P}_{\mathrm{N}} / \mathrm{C}_{\mathrm{I}}\right)$. Medidas da emissão de fluorescência da clorofila foram realizadas com um fluorômetro modulado 6400-40 LCF, (LI-COR, Inc., Lincoln, EUA), integrado ao LI-6400F. A fluorescência mínima $\left(\mathrm{F}_{\mathrm{O}}\right)$ e a máxima $\left(\mathrm{F}_{\mathrm{M}}\right)$ foram monitoradas em tecidos foliares adaptados ao escuro (30 min) e a fluorescência instantânea $\left(\mathrm{F}_{\mathrm{S}}\right)$ e máxima $\left(\mathrm{F}_{\mathrm{M}}{ }^{\prime}\right)$ em tecidos adaptados à luminosidade, pelo método do pulso de saturação $\left(\lambda=630 \mathrm{~nm}, \mathrm{Q} \sim 6000 \mu \mathrm{mol} \mathrm{m} \mathrm{m}^{-2} \mathrm{~s}^{-1}, 0,8 \mathrm{~s}\right)$. A fluorescência basal $\left(F_{O}{ }^{\prime}\right)$ foi medida após a excitação do fotossistema I com radiação vermelha-distante $\left(\lambda=740 \mathrm{~nm}, \mathrm{Q} \sim 5 \mu \mathrm{mol} \mathrm{m}{ }^{-2} \mathrm{~s}^{-1}, 2,0 \mathrm{~s}\right)$. Com base nesses sinais, foram calculadas a fluorescência variável no escuro $\left(F_{V}=F_{M}-F_{O}\right)$ e na luz $\left(\Delta F=F_{M}{ }^{\prime}-F_{S}\right)$ e algumas variáveis fotoquímicas: eficiência quântica potencial $\left(\mathrm{F}_{\mathrm{V}} / \mathrm{F}_{\mathrm{M}}\right)$ e efetiva $\left(\Delta \mathrm{F} / \mathrm{F}_{\mathrm{M}}{ }^{\prime}\right)$ do fotossistema II (FSII); transporte aparente de elétrons $\left[\mathrm{ETR}=(\mathrm{Q} \times \Delta \mathrm{F}) / \mathrm{F}_{\mathrm{M}}{ }^{\prime} \mathrm{x}\right.$ $0,5 \times 0,84]$; e coeficientes de extinção não-fotoquímica $\left[\mathrm{NPQ}=\left(\mathrm{F}_{\mathrm{M}}-\mathrm{F}_{\mathrm{M}}{ }^{\prime}\right) / \mathrm{F}_{\mathrm{M}}{ }^{\prime}\right]$ e fotoquímica $\left[\mathrm{q}_{\mathrm{P}}=\left(\mathrm{F}_{\mathrm{M}}{ }^{\prime}-\mathrm{F}_{\mathrm{S}}\right) /\right.$ $\left.\left(F_{M}{ }^{\prime}-F_{O}{ }^{\prime}\right)\right]$ da fluorescência (Rohacek, 2002). A relação $E T R / P_{N}$ foi utilizada para a estimativa do dreno alternativo de elétrons (Ribeiro et al., 2009).

O potencial da água na folha foi medido na antemanhã $\left(\Psi_{5 \mathrm{~h}}\right)$ e às $13 \mathrm{~h}\left(\Psi_{13 \mathrm{~h}}\right)$ com câmara de pressão modelo 3005 (Soil Moisture Equipment Corp., Santa Barbara, EUA). A condutância hidráulica aparente das plantas $\left(\mathrm{K}_{\mathrm{L}}\right)$ foi calculada conforme descrito por Pinheiro et al. (2005): $\mathrm{K}_{\mathrm{L}}=\left\{\left(\mathrm{g}_{\mathrm{S}} \times \mathrm{DPV} \mathrm{L}\right) /\left[\left(\Psi_{5 \mathrm{~h}}-\Psi_{13 \mathrm{~h}}\right)\right.\right.$ Patm $\left.]\right\}$, em que $\mathrm{DPV}_{\mathrm{L}}$ representa a diferença de pressão de vapor entre folha e ar e Patm é a pressão atmosférica local.

Determinações dos teores de clorofila a e b e carotenóides $(\mathrm{x}+\mathrm{c})$ foram feitas em discos foliares de $0,9 \mathrm{~cm}$ de diâmetro. Os discos de massa fresca foram macerados com a adição de $5 \mathrm{~mL}$ de acetona a $80 \%$, $\mathrm{v} / \mathrm{v}$, e o almofariz foi mantido resfriado em ambiente escurecido. Após centrifugação, a absorbância do sobrenadante foi avaliada em espectrofotômetro a 470, 646 e $663 \mathrm{~nm}$. Os cálculos da concentração de pigmentos foram realizados conforme Lichtenthaler \& Wellburn (1983).

Na determinação dos teores foliares de carboidratos, foram considerados os açúcares solúveis totais (AS), sacarose (Sac) e amido (AM), em amostras coletadas às $13 \mathrm{~h}$. $\mathrm{O}$ teor de carboidrato total não estrutural (AT) foi determinado indiretamente, sendo $\mathrm{AT}=\mathrm{AS}+\mathrm{AM}$. A extração e a purificação dos açúcares solúveis para determinação de AS e Sac foram realizadas com uma solução de metanol:clorofórmio:água (MCW, 12:5:3, v/v) segundo Bielesk \& Turner (1966). A quantificação de Sac e AS foi realizada pelos métodos descritos por Dubois et al. (1956) e Handel (1968), com leituras de absorbância a $490 \mathrm{~nm}$. A determinação de AM foi realizada segundo o método enzimático descrito por Amaral et al. (2007), com a concentração de glicose determinada em leitor de microplacas modelo EL307C (Bio-Tek Instruments, Inc., Winooski, EUA), em 490 nm.

A partição de matéria seca foi avaliada pela separação das plantas nas frações folha, caule e raiz, sendo essas coletadas e desidratadas em estufa de circulação forçada a $60^{\circ} \mathrm{C}$ até a obtenção da massa constante. O teor foliar dos macronutrientes $\mathrm{N}, \mathrm{P}, \mathrm{K}, \mathrm{S}$, $\mathrm{Ca}$ e $\mathrm{Mg}$, foi avaliado conforme os métodos descritos por Bataglia et al. (1983).

O arranjo experimental foi o de blocos ao acaso e os dados foram submetidos à análise de variância, com as médias de quatro repetições comparadas pelo teste de Tukey, a 5\% de probabilidade.

\section{Resultados e Discussão}

A análise nutricional das plantas revelou alterações significativas nos teores foliares de $\mathrm{N}, \mathrm{Ca}, \mathrm{Mg}$ e $\mathrm{P}$ de acordo com os tratamentos (Tabela 1). As plantas do tratamento P- apresentaram teores de $\mathrm{P}$ com cerca da metade do valor observado no tratamento PA. No tratamento $\mathrm{P}+$, o teor foliar de $\mathrm{P}$ foi cerca de 1,5 vezes maior em relação ao tratamento $\mathrm{PA}$. $\mathrm{O}$ incremento no teor foliar de $\mathrm{Mg}$ e a redução no teor de $\mathrm{N}$ nas plantas do tratamento $\mathrm{P}+$ confirmam a interação sinergística entre $\mathrm{Mg}$ e $\mathrm{P}$ e a antagônica entre $\mathrm{N}$ e P (Menard \& Malavolta, 1962). Embora tenham apresentado os menores teores foliares de $\mathrm{N}$ em relação aos demais tratamentos, as plantas $\mathrm{P}+$ não exibiram teores limitantes desse nutriente (Clemente et al., 2008). Os maiores teores de $\mathrm{Ca}$ nas folhas foram observados no tratamento P-, e foram cerca de $13 \%$ e $24 \%$ maiores em relação aos tratamentos $\mathrm{PA}$ e $\mathrm{P}+$, respectivamente. Com exceção de $\mathrm{P}$, pode-se considerar que não houve variação nos teores de nutrientes que induzisse resposta biológica diferencial, com $\mathrm{N}, \mathrm{K}, \mathrm{Ca}, \mathrm{Mg}$ e $\mathrm{S}$ próximos ou acima dos teores recomendados para cafeeiros 
jovens (Clemente et al., 2008), independentemente do tratamento.

O potencial de água na folha não diferiu entre os tratamentos (Figura $1 \mathrm{~A}$ ), considerando tanto as medidas realizadas na antemanhã $\left(\Psi_{5 \mathrm{~h}}\right)$ como às 13 $\mathrm{h}\left(\Psi_{13 \mathrm{~h}}\right)$. Os cafeeiros $\mathrm{P}+$ apresentaram maior $\mathrm{K}_{\mathrm{L}} \mathrm{em}$ relação ao tratamento $\mathrm{PA}$, e os menores valores foram observados nas plantas do tratamento P- (Figura $1 \mathrm{~B}$ ). A menor condutância estomática $\left(\mathrm{g}_{\mathrm{s}}\right)$ das plantas de P(Figura $2 \mathrm{~B}$ ) está associada ao baixo $\mathrm{K}_{\mathrm{L}}$ observado nesse tratamento (Figura $1 \mathrm{~B}$ ). Há estreita relação entre $\mathrm{g}_{\mathrm{S}} \mathrm{e}$ $\mathrm{K}_{\mathrm{L}}$, e pode-se observar que as plantas com alto $\mathrm{K}_{\mathrm{L}}$ são mais sensíveis à cavitação e apresentam fechamento estomático em valores mais altos de $\Psi$, em condição limitante (Schultz, 2003). Mesmo sob a constatação de que as plantas do tratamento PA apresentaram menor $\mathrm{K}_{\mathrm{L}}$ do que as do tratamento $\mathrm{P}+$, os valores dessa variável estão dentro de uma faixa considerada normal por Pinheiro et al. (2005), para cafeeiros conduzidos com boa disponibilidade de água.

Sabe-se que o fluxo de água nas plantas é mediado por proteínas intrínsecas das membranas plasmáticas denominadas aquaporinas. A atividade dessas proteínas, responsáveis pelo aumento e redução da resistência ao fluxo da água na planta, regula a absorção de água do solo e também a condutância hidráulica da planta (Kramer \& Boyer, 1995). Considerando-se que as aquaporinas precisam ser fosforiladas para ficarem ativas (Tournaire-Roux et al., 2003), sugere-se que a maior disponibilidade de $\mathrm{P}$ permitiu maior atividade de aquaporinas, o que justificaria maiores $\mathrm{K}_{\mathrm{L}}$ nas plantas do tratamento $\mathrm{P}+$.

Além de causar menor $\mathrm{g}_{\mathrm{s}}$ (Figura $2 \mathrm{~B}$ ), a deficiência de $\mathrm{P}$ também afetou negativamente a assimilação

Tabela 1. Teores foliares $\left(\mathrm{g} \mathrm{kg}^{-1}\right)^{(1)}$ de macronutrientes em cafeeiros jovens cultivados em casa-de-vegetação, em solos sem adição de fósforo (P-), com adição recomendada do nutriente $(\mathrm{PA})$ e com o dobro da dose recomendada $(\mathrm{P}+)^{(2)}$.

\begin{tabular}{lrrr}
\hline Nutriente & \multicolumn{1}{c}{$\mathrm{P}-$} & \multicolumn{1}{c}{$\mathrm{PA}$} & \multicolumn{1}{c}{$\mathrm{P}+$} \\
\hline $\mathrm{N}$ & $48,80 \pm 1,04 \mathrm{a}$ & $46,40 \pm 0,44 \mathrm{a}$ & $41,93 \pm 1,96 \mathrm{~b}$ \\
$\mathrm{P}$ & $0,60 \pm 0,01 \mathrm{c}$ & $1,23 \pm 0,15 \mathrm{~b}$ & $1,87 \pm 0,06 \mathrm{a}$ \\
$\mathrm{K}$ & $19,70 \pm 1,82 \mathrm{a}$ & $20,17 \pm 0,50 \mathrm{a}$ & $22,67 \pm 0,15 \mathrm{a}$ \\
$\mathrm{Ca}$ & $16,20 \pm 0,10 \mathrm{a}$ & $14,13 \pm 1,59 \mathrm{~b}$ & $12,23 \pm 0,38 \mathrm{~b}$ \\
$\mathrm{Mg}$ & $4,13 \pm 0,32 \mathrm{~b}$ & $4,63 \pm 0,47 \mathrm{~b}$ & $6,27 \pm 0,06 \mathrm{a}$ \\
$\mathrm{S}$ & $2,43 \pm 0,40 \mathrm{a}$ & $2,50 \pm 0,17 \mathrm{a}$ & $2,60 \pm 0,01 \mathrm{a}$ \\
\hline
\end{tabular}

(1)Avaliação realizada 70 dias após a aplicação dos tratamentos. ${ }^{(2)}$ Médias \pm desvio-padrão seguidas de letras iguais não diferem entre si, pelo teste de Tukey a $5 \%$ de probabilidade. de $\mathrm{CO}_{2}\left(\mathrm{P}_{\mathrm{N}}\right)$, quando comparada aos tratamentos tratamentos $\mathrm{PA}$ e $\mathrm{P}+$ (Figura $2 \mathrm{~A}$ ). Por sua vez, o tratamento $\mathrm{P}+$ fez com que os valores de $\mathrm{g}_{\mathrm{s}}$ fossem ligeiramente superiores em relação ao tratamento PA, sendo essa diferença intensificada às $13 \mathrm{~h}$ (Figura $2 \mathrm{~B}$ ). Esse padrão de resposta de $g_{S}$ à adubação fosfatada foi observado também em $\mathrm{P}_{\mathrm{N}}$, o que fez com que as plantas do tratamento $\mathrm{P}+$ apresentassem maior fotossíntese durante todo o período de avaliação (Figura 2 A e B).

Em relação à hidratação dos tecidos, as plantas apresentavam valores similares de $\Psi_{13 \mathrm{~h}}$ (Figura $1 \mathrm{~A}$ ) e, portanto, a variação de $g_{s}$ não teria sido ocasionada por sinal hidráulico. Todavia, a significativa relação entre os valores máximos de $g_{\mathrm{s}}$ e os de $\mathrm{K}_{\mathrm{L}}(\mathrm{r}=0,992$, $\mathrm{p}<0,076)$ sugere a sinalização hidráulica. De fato, Schultz (2003) relata que, além dos aspectos hidráulicos, a abertura estomática é regulada por sinais químicos e hormonais. A similaridade entre os tratamentos, quando

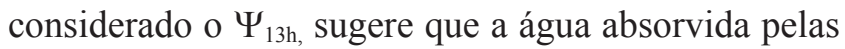
plantas foi suficiente para manter a hidratação das plantas de P-. Esse aspecto foi regulado também pela menor transpiração resultante da menor área foliar e $g_{S}$ (Figura 2 B), nesse tratamento.

A menor produção de ATP poderia afetar indiretamente $\mathrm{g}_{\mathrm{s}}$ (Lawlor \& Cornic, 2002); todavia, essa relação justificaria apenas a menor abertura estomática nas plantas em P- (Figura 2 B). Uma alternativa plausível seria a regulação da abertura estomática relacionada à variação da concentração de $\mathrm{CO}_{2}$ intercelular $\left(\mathrm{C}_{\mathrm{I}}\right)$, de forma a estimular a abertura estomática pela redução de $C_{I}$ ou vice-versa (Lambers et al., 1998). Neste

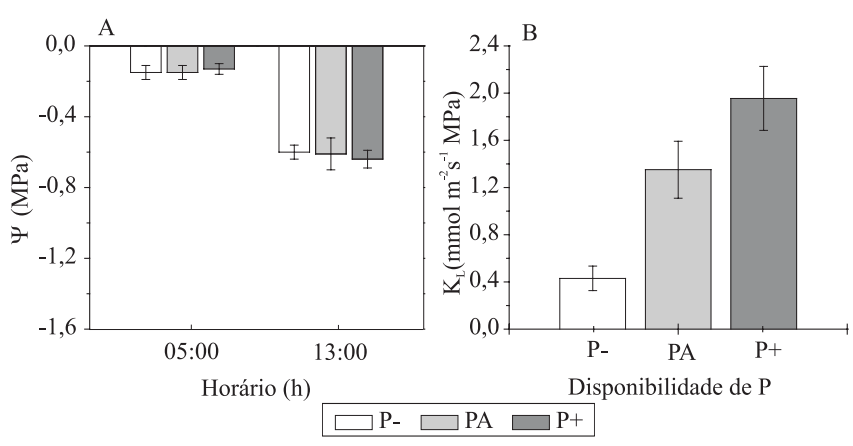

Figura 1. Potencial da água na folha $(\Psi)$, medido às 5:00 h e às 13:00 h (A), e condutância hidráulica da planta $(\mathrm{B}) \mathrm{em}$ cafeeiros jovens cultivados por 70 dias em solos sem adição de fósforo (P-), e com adição recomendada do nutriente (PA) e duas vezes superior à recomendada $(\mathrm{P}+)$. Cada histograma representa média \pm desvio-padrão de quatro repetições. 

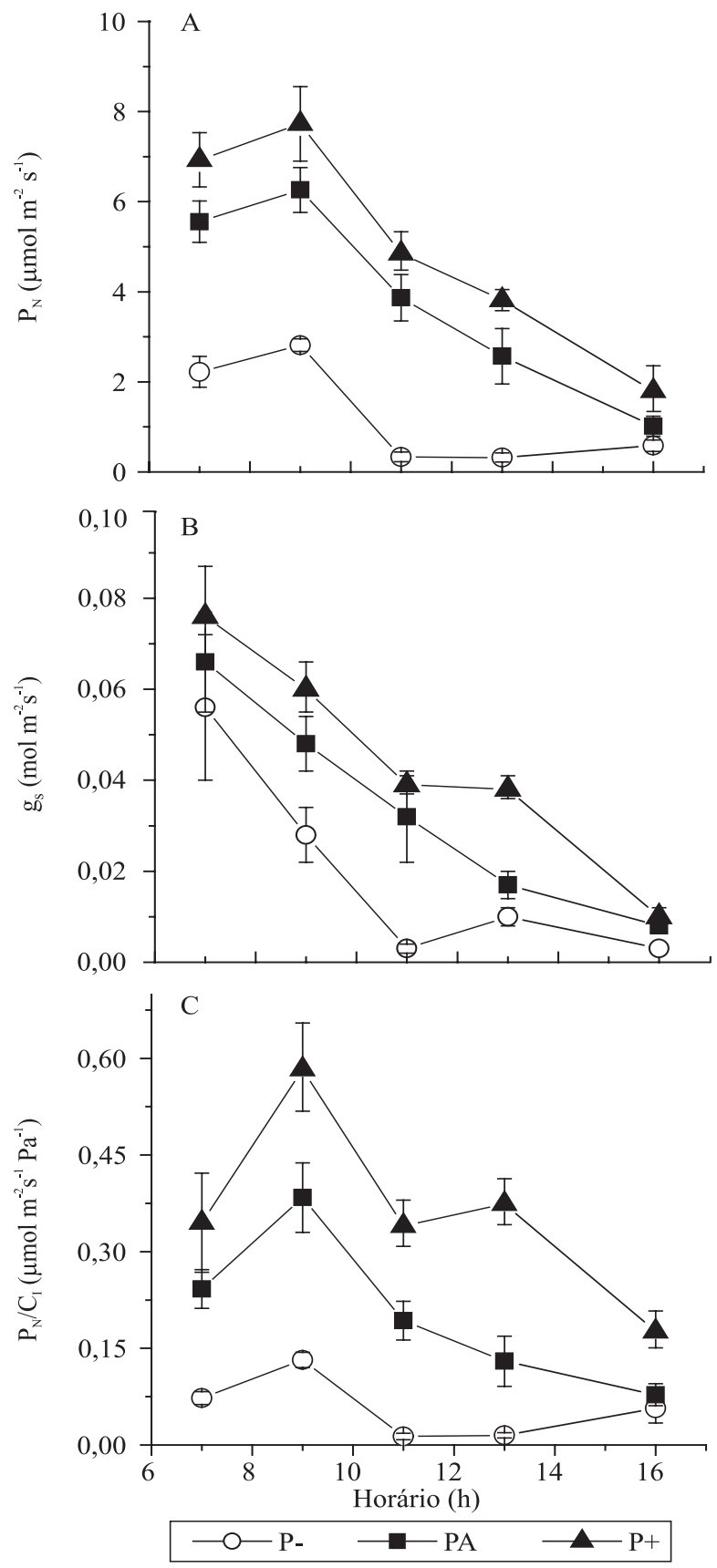

Figura 2. Variação diurna da assimilação de $\mathrm{CO}_{2}$ $\left(\mathrm{P}_{\mathrm{N}}, \mathrm{A}\right)$ condutância estomática $\left(\mathrm{g}_{\mathrm{S}}, \mathrm{B}\right)$ e eficiência instantânea de carboxilação $\left(\mathrm{P}_{\mathrm{N}} / \mathrm{C}_{\mathrm{I}}, \mathrm{C}\right)$, em cafeeiros jovens cultivados por 70 dias em solos sem adição de fósforo (P-), e com adição recomendada do nutriente $(\mathrm{PA})$ e duas vezes a recomendada $(\mathrm{P}+)$. Cada símbolo representa a média \pm desvio-padrão de quatro repetições. Medidas realizadas entre 7:00 e 16:00 h, com variação da temperatura do ar entre 26,4 e $36,2^{\circ} \mathrm{C}$, radiação fotossinteticamente ativa máxima de $1.600 \mu \mathrm{mol} \mathrm{m}^{-2} \mathrm{~s}^{-1} \mathrm{e}$ déficit de pressão de vapor entre a folha e o ar variando entre 1,8 e $3,4 \mathrm{kPa}$. estudo, a maior $\mathrm{g}_{\mathrm{S}}$ nas plantas $\mathrm{P}+$ pode ser explicada pelos menores valores de $C_{I}$ neste tratamento, quando comparado ao $\mathrm{PA}\left(148 \pm 6\right.$ vs. $\left.201 \pm 8 \mu \mathrm{mol} \mathrm{mol}^{-1}\right)$.

A maior disponibilidade de $\mathrm{P}$ também resultou no incremento médio de $35 \%$ em $\mathrm{P}_{\mathrm{N}} / \mathrm{C}_{\mathrm{I}}$ (Figura 2 C), quando comparado ao tratamento PA. Este incremento sugere que o melhor desempenho bioquímico é uma das causas aparentes do aumento em $\mathrm{P}_{\mathrm{N}}$. Sabe-se que a disponibilidade de $\mathrm{P}$ afeta diretamente a ativação de algumas enzimas do ciclo de Calvin-Benson, tais como a ribulose-1,5-bisfosfato carboxilase/oxigenase, frutose-1,6-bisfosfatase e a sedoheptulose-1,7-bisfosfatase (Shubhra et al., 2004). Todavia, as plantas PA já exibiam teores foliares adequados de $\mathrm{P}$ e, portanto, a atividade das enzimas do ciclo $\mathrm{C} 3$ não deveria ser limitante em relação ao tratamento $\mathrm{P}+$. $\mathrm{O}$ melhor desempenho bioquímico no tratamento $\mathrm{P}+$ poderia, dessa forma, ser consequência do melhor estado metabólico da planta, o que pode ser uma resposta da fonte estimulada pela maior demanda por fotoassimilados nos drenos (Iglesias et al., 2002).

As plantas $\mathrm{P}+$ apresentaram maior crescimento, porém, estudos mais detalhados sobre a interação entre a bioquímica da fotossíntese e o excesso de P devem ser realizados a fim de esclarecer uma provável interação. Quanto ao tratamento $\mathrm{P}$-, os efeitos da ausência de $\mathrm{P}$ foram evidentes e confirmaram os dados da literatura acerca da redução da fotossíntese em plantas deficientes devido a problemas de origem bioquímica (Hendrickson et al., 2004; Shubhra et al., 2004).

A deficiência de fósforo também reduziu significativamente a eficiência quantica potencial, $\mathrm{F}_{\mathrm{V}} / \mathrm{F}_{\mathrm{M}}$, o que indica ocorrência de dano fotoquímico (Tabela 2). Esse dano pode ter sido consequência direta da ausência de $\mathrm{P}$ e também do excesso de energia no fotossistema II, uma vez que as plantas apresentavam redução significativa em $\mathrm{P}_{\mathrm{N}}$ (Figura 2 A). Não houve variação significativa de $F_{V} / F_{M}$ entre plantas do tratamento $\mathrm{PA}$ e $\mathrm{P}+$. Contudo, observou-se $\Delta \mathrm{F} / \mathrm{F}_{\mathrm{M}}{ }^{\prime}$ maior nas plantas do tratamento $\mathrm{P}+\mathrm{e}$ menor no tratamento $\mathrm{P}$-.

Ao se considerar a relação entre a atividade fotoquímica e bioquímica, pode-se sugerir que o aumento em $\mathrm{P}_{\mathrm{N}} / \mathrm{C}_{\mathrm{I}}$ no tratamento $\mathrm{P}+$ (Figura $2 \mathrm{C}$ ) causou maior consumo de ATP e NADPH, o que poderia estimular ("up-regulation") a atividade fotoquímica (Shubhra et al., 2004). No tratamento $\mathrm{P}+$, os maiores valores de $\Delta \mathrm{F} / \mathrm{F}_{\mathrm{M}}{ }^{\prime}$ determinaram maior 
ETR, com aumentos de 45 e $85 \%$ em relação aos tratamentos PA e P-, respectivamente (Tabela 2). Além disso, NPQ aumentou significativamente nas plantas do tratamento P-. Essa via de dissipação de energia ocorre em consequência da formação de um gradiente de $\mathrm{pH}$ transmembranar nos tilacóides dos cloroplastos e está relacionada à maior atividade do ciclo das xantofilas (Critchley, 1998). Os maiores valores de NPQ nas plantas P- estão associados à dissipação do excesso de energia de excitação originado pelo menor consumo de elétrons por processos assimilatórios como a fotossíntese.

Em relação ao coeficiente de extinção fotoquímica $\left(\mathrm{q}_{\mathrm{P}}\right)$, os maiores valores foram observados nas plantas do tratamento $\mathrm{P}+$, o que indica maior extinção da fluorescência por processos relacionados ao transporte de elétrons nos cloroplastos e consequente geração de ATP e NADPH. Este melhor desempenho fotoquímico ocorreu mesmo sem alterações significativas do NPQ em relação às plantas $\mathrm{PA}$ e $\mathrm{P}+$, o que atende à demanda por produtos fotoquímicos devido à alta atividade fotossintética no tratamento com a maior dose de $\mathrm{P}$ (Figura $2 \mathrm{~A}$ ).

A relação $\mathrm{ETR} / \mathrm{P}_{\mathrm{N}}$, utilizada para estimar a atividade de drenos alternativos de elétrons (Ribeiro et al., 2009), foi significativamente maior no tratamento P(Tabela 2). Este aumento indica maior utilização de energia fotoquímica em outros processos metabólicos que não a fixação de $\mathrm{CO}_{2}$ (Baker, 2008), e evidencia a menor eficiência fotossintética das plantas P-.

Tabela 2. Eficiência quântica potencial $\left(\mathrm{F}_{\mathrm{V}} / \mathrm{F}_{\mathrm{M}}\right)$ e efetiva $\left(\Delta \mathrm{F} / \mathrm{F}_{\mathrm{M}}{ }^{\prime}\right)$ do fotossistema II, coeficiente de extinção fotoquímico $\left(\mathrm{q}_{\mathrm{P}}\right)$ e não-fotoquímico (NPQ) da fluorescência, transporte aparente de elétrons (ETR) e relação entre ETR e a assimilação de $\mathrm{CO}_{2}\left(E T R / \mathrm{P}_{\mathrm{N}}\right)$, em cafeeiros jovens ${ }^{(1)}$ cultivados em solos sem adição de fósforo (P-), e com adição recomendada do nutriente (PA) e duas vezes superior à recomendada $(\mathrm{P}+)^{(2)}$.

\begin{tabular}{lccc}
\hline Variáveis $^{(3)}$ & $\mathrm{P}-$ & $\mathrm{PA}$ & $\mathrm{P}+$ \\
\hline $\mathrm{F}_{\mathrm{V}} / \mathrm{F}_{\mathrm{M}}$ & $0,244 \pm 0,023 \mathrm{~b}$ & $0,673 \pm 0,021 \mathrm{a}$ & $0,735 \pm 0,044 \mathrm{a}$ \\
$\Delta \mathrm{F} / \mathrm{F}_{\mathrm{M}}{ }^{\prime}$ & $0,017 \pm 0,004 \mathrm{c}$ & $0,055 \pm 0,008 \mathrm{~b}$ & $0,095 \pm 0,009 \mathrm{a}$ \\
$\mathrm{q}_{\mathrm{P}}$ & $0,138 \pm 0,044 \mathrm{c}$ & $0,212 \pm 0,036 \mathrm{~b}$ & $0,329 \pm 0,031 \mathrm{a}$ \\
$\mathrm{NPQ}$ & $4,62 \pm 0,31 \mathrm{a}$ & $3,87 \pm 0,10 \mathrm{~b}$ & $3,70 \pm 0,09 \mathrm{~b}$ \\
$\mathrm{ETR}$ & $10,2 \pm 3,3 \mathrm{c}$ & $34,4 \pm 5,2 \mathrm{~b}$ & $59,9 \pm 6,0 \mathrm{a}$ \\
ETR $/ \mathrm{P}_{\mathrm{N}}$ & $32,0 \pm 9,0 \mathrm{a}$ & $9,3 \pm 2,6 \mathrm{~b}$ & $12,6 \pm 0,6 \mathrm{~b}$ \\
\hline
\end{tabular}

${ }^{(1)}$ Avaliações realizadas 70 dias após a adubação fosfatada. ${ }^{(2)}$ Médias \pm desvio-padrão seguidas de letras iguais não diferem entre si, pelo teste de Tukey a $5 \%$ de probabilidade. ${ }^{(3)}$ Horários de medição: $F_{V} / F_{M}$ às 15:00 h; demais variáveis às 11:00 $\mathrm{h}$.
Em relação aos tratamentos $\mathrm{PA}$ e $\mathrm{P}+$, não houve variação significativa de ETR $/ \mathrm{P}_{\mathrm{N}}$.

Houve redução nos teores de pigmentos fotossintéticos nas plantas do tratamento P- (Figura $3 \mathrm{~A}$ ). Esta resposta está associada à menor captação de luz pelo FSII, com consequente redução de ETR (Lawlor \& Cornic, 2002). Apesar de as plantas do tratamento PA apresentarem menores valores de teor de pigmentos em relação ao tratamento $\mathrm{P}+$, estes se encontram compatíveis com os valores relatados por Torres Netto et al. (2005) em cafeeiros. Em relação ao aspecto nutricional, torna-se importante considerar que houve incremento significativo no teor foliar de $\mathrm{Mg}$ nas plantas $\mathrm{P}+$ (Tabela 1). Uma vez que esse elemento compõe as moléculas de clorofila, o maior teor foliar de $\mathrm{Mg}$ poderia ser uma das causas dos maiores teores de clorofila nas plantas $\mathrm{P}+$.

$\mathrm{O}$ tratamento $\mathrm{P}+$ causou incremento significativo no teor de sacarose (Sac) e açúcares solúveis (AS) em folhas de cafeeiros jovens, com redução nos teores de amido (AM) (Figura 3 B). Como a ciclagem de P celular é determinada pela síntese de sacarose no citossol e de amido no estroma do cloroplasto (Hendrickson et al., 2004), pode-se considerar que o aumento em $\mathrm{P}_{\mathrm{N}}$ (Figura 2 A) concomitante ao incremento de $\mathrm{Sac}$ e a redução de AM (Figura $3 \mathrm{~B}$ ) indicariam maior ciclagem de P. Essa alteração metabólica, mediada por variações nos teores de carboidratos, pode ter estimulado $\mathrm{P}_{\mathrm{N}}$ (Iglesias et al., 2002) nas plantas cultivadas em solos

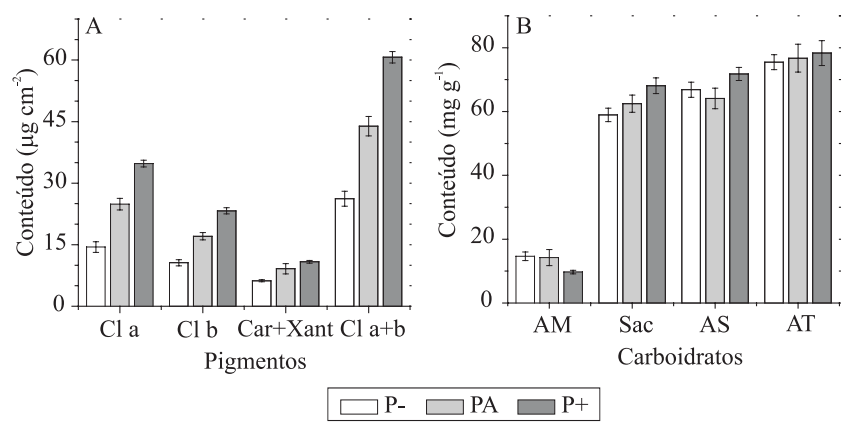

Figura 3. Teores de clorofila $a(\mathrm{Cl} a), b(\mathrm{Cl} b), a+b$ $(\mathrm{Cl} \mathrm{a}+\mathrm{b})$, carotenóides e xantofilas (Car+Xant) (A), e de amido (AM), sacarose (Sac), açúcares solúveis (AS) e carboidratos totais não estruturais (AT) (B), em folhas de cafeeiros jovens cultivados por 70 dias em solos sem adição de fósforo (P-), e com adição recomendada do nutriente (PA) e duas vezes a recomendada $(\mathrm{P}+)$. Cada histograma representa média \pm desviopadrão de quatro repetições. Coletas das folhas realizadas às 5:00 $\mathrm{h}$ (pigmentos) e às 13:00 $\mathrm{h}$ (carboidratos). 
com maior concentração de P. A falta de P limitou a síntese de Sac (Figura 3 B), uma vez que a triose-P requerida na síntese desse dissacarídeo deve ser exportada pelo cloroplasto, processo que é dependente da disponibilidade de $\mathrm{P}$ no citossol celular (Hendrickson et al., 2004).

Os valores de hexoses dados pela diferença entre AS e Sac estão ligeiramente abaixo do observado por DaMatta et al. (2008), em C. arabica com dez anos de idade, e por Silva et al. (2010), em mudas de $C$. canephora, ao passo que os teores de amido e sacarose foram bem superiores no presente estudo. Evidentemente, tais variações estão relacionadas à diferença de idade entre as plantas (mudas e árvores) e também à variação genética intra e interespecífica.

Houve maior crescimento das plantas com a maior disponibilidade de $\mathrm{P}$ (Tabela 3), tendo-se observado variação significativa na matéria seca de folhas $(\triangle \mathrm{MSF})$, caule ( $\triangle \mathrm{MSC})$ e raízes ( $\triangle \mathrm{MSR})$. Em contrapartida, as plantas do tratamento $\mathrm{P}$ - apresentaram os menores valores de $\triangle \mathrm{MSF}, \triangle \mathrm{MSC}$ e $\triangle \mathrm{MSR}$ após 70 dias do início do tratamento. $\mathrm{O}$ mesmo efeito foi constatado na relação raiz/parte aérea, a qual foi reduzida, nas plantas do tratamento P- em relação aos tratamentos $\mathrm{PA}$ e $\mathrm{P}+$. Essa resposta ocorreu pelo fato da inibição do crescimento motivada pela ausência de P ter sido maior nas raízes do que na parte aérea.

Mesmo reduzida pela ausência de $\mathrm{P}$, a relação raiz/ parte aérea foi superior à relatada na literatura, i.e., 0,43 para 0,22 (Silva et al., 2010). A matéria seca total das plantas aumentou significativamente com a maior dose de P (Tabela 3). O maior crescimento das plantas

Tabela 3. Variação da matéria seca de folhas $\triangle \mathrm{MSF}$ ), do caule ( $\triangle \mathrm{MSC})$, das raízes ( $\triangle \mathrm{MSR})$ e total $(\triangle \mathrm{MST})$, e relação $\mathrm{MSR} /(\mathrm{MSC}+\mathrm{MSF})$ de cafeeiros jovens ${ }^{(1)}$ cultivados em solos sem adição de fósforo (P-), e com adição recomendada do nutriente $(\mathrm{PA}) \mathrm{e}$ duas vezes superior à recomendada $(\mathrm{P}+)^{(2)}$.

\begin{tabular}{lccc}
\hline Variáveis & $\mathrm{P}-$ & $\mathrm{PA}$ & $\mathrm{P}+$ \\
\hline$\Delta$ MSF $(\mathrm{g})$ & $1,88 \pm 0,20 \mathrm{c}$ & $3,20 \pm 0,13 \mathrm{~b}$ & $3,75 \pm 0,19 \mathrm{a}$ \\
$\Delta \mathrm{MSC}(\mathrm{g})$ & $1,12 \pm 0,11 \mathrm{c}$ & $3,15 \pm 0,10 \mathrm{~b}$ & $3,59 \pm 0,13 \mathrm{a}$ \\
$\Delta \mathrm{MSR}(\mathrm{g})$ & $0,79 \pm 0,09 \mathrm{c}$ & $1,99 \pm 0,08 \mathrm{~b}$ & $2,17 \pm 0,07 \mathrm{a}$ \\
$\Delta \mathrm{MST}(\mathrm{g})$ & $3,78 \pm 0,11 \mathrm{c}$ & $8,33 \pm 0,19 \mathrm{~b}$ & $9,51 \pm 0,20 \mathrm{a}$ \\
MSR/(MSC+MSF) & $0,43 \pm 0,09 \mathrm{~b}$ & $0,62 \pm 0,05 \mathrm{a}$ & $0,58 \pm 0,02 \mathrm{a}$ \\
\hline
\end{tabular}

${ }^{(1)}$ Avaliação realizada 70 dias após a adubação fosfatada. ${ }^{(2)}$ Médias \pm desviopadrão de tratamentos seguidas de letras iguais diferem entre si, pelo teste de Tukey a $5 \%$ de probabilidade. do tratamento $\mathrm{P}+$ esteve relacionado ao maior teor de carboidratos solúveis nas folhas (Figura $3 \mathrm{~B}$ ) que, por sua vez, foi consequência do maior $\mathrm{P}_{\mathrm{N}}$ (Figura $2 \mathrm{~A}$ ).

Uma questão que deve ser observada é a de que a recomendação de adubação fosfatada não leva em consideração a eficiência diferencial que as cultivares de cafeeiros podem apresentar em relação ao uso de determinado nutriente (Pozza et al., 2009). Além disso, é razoável reconhecer que os programas de melhoramento genético tenham selecionado ao longo do tempo genótipos cuja exigência nutricional seja diferente (Fazuoli et al., 2002), o que justificaria a resposta significativa e positiva ao aumento na adubação fosfatada.

\section{Conclusões}

1. A baixa disponibilidade de fósforo no solo compromete o crescimento de mudas do cafeeiro em condições de boa disponibilidade hídrica, em razão da disfunção no metabolismo fotossintético por limitações de origem estomática e metabólica.

2. A maior disponibilidade de fósforo no solo em relação aos níveis recomendados promove melhor desempenho fotossintético, induzido pela maior abertura estomática, e maior atividade bioquímica e fotoquímica de mudas do cafeeiro, que apresentam, dessa forma, maior disponibilidade de energia, na forma de carboidratos, para o crescimento.

\section{Referências}

AMARAL, L.I.V. do; GASPAR, M.; COSTA, P.M.F.; AIDAR, M.P.M.; BUCKERIDGE, M.S. Novo método enzimático rápido e sensível de extração e dosagem de amido em materiais vegetais. Hoehnea, v.34, p.425-431, 2007.

BAKER, N.R. Chlorophyll fluorescence: a probe of photosynthesis in vivo. Annual Review of Plant Biology, v.59, p.89-113, 2008.

BATAGLIA, O.C.; FURLANI, A.M.C.; TEIXEIRA, J.P.F.; FURLANI, P.R.; GALLO, J.R. Métodos de análise química de plantas. Campinas: IAC, 1983. 48p. (IAC. Boletim técnico, 78).

BIELESKI, R.L.; TURNER, N.A. Separation and estimation of amino acids in crude plant extracts by thin-layer electrophoresis and chromatography. Analytical Biochemistry, v.17, p.278-293, 1966.

CLEMENTE, F.M.V.T.; CARVALHO, J.G. de; GUIMARÃES, R.J.; MENDES, A.N.G. Faixas críticas de teores foliares de macronutrientes no cafeeiro em pós-plantio - primeiro ano. Coffee Science, v.3, p.47-57, 2008. 
CRITCHLEY, C. Photoinhibition. In: RAGHAVENDRA, A.S. Photosynthesis: a comprehensive treatise. Cambridge: Cambridge University, 1998. p.264-272.

DUBOIS, M.; GILLES, K.A.; HAMILTON, J.K.; REBERS, P.A.; SMITH, F. Colorimetric method for determination of sugars and related substances. Analytical Chemistry, v.28, p.350-356, 1956.

FAZUOLI, L.C.; GALLO, P.B.; CERVELLINI, G.S.; BARROS, I. de; RAIJ, B. van. Café - Coffea arabica L. In: FAHL, J.I.; CAMARGO, M.B.P. de; PIZZINATTO, M.A.; BETTI, J.A.; MELO, A.M.T. de; DE MARIA, I.C.; FURLANI, A.M.C. Instruções agrícolas para as principais culturas econômicas. 6.ed. rev. atual. Campinas: IAC, 1998. p.59-63. (IAC. Boletim, 200).

FAZUOLI, L.C.; MEDINA FILHO, H.P.; GONÇALVES, W.; GUERREIRO FILHO, O.; SILVAROLLA, M.B. Melhoramento do cafeeiro: variedades tipo arábicas obtidas no Instituto Agronômico de Campinas. In: ZAMBOLIM, L. (Ed.). O estado da arte de tecnologias na produção de café. Viçosa: UFV, 2002. p.163-215.

DAMATTA, F.M.; CUNHA, R.L.; ANTUNES, W.C.; MARTINS, S.C.;ARAÚJO,W.L.;FERNIE,A.R.;MORAES, G.A.Infield-grown coffee trees source-sink manipulation alters photosynthetic rates, independently of carbon metabolism, via alterations in stomatal function. New Phytologist, v.178, p.348-357, 2008.

FLÜGGE, U.I.; HÄUSLER, R.E.; LUDEWIG, F.; FISCHER, K. Functional genomics of phosphate antiport systems of plastids. Physiologia Plantarum, v.118, p.475-482, 2003.

HANDEL, E. van. Direct microdetermination of sucrose. Analytical Biochemistry, v.22, p.280-283, 1968.

HENDRICKSON, L.; CHOW, W.S.; FURBANK, R.T. Low temperature effects on grapevine photosynthesis: the role of inorganic phosphate. Functional Plant Biology, v.31, p.789-801, 2004.

IGLESIAS, D.J.; LLISO, I.; TADEO, F.R.; TALON, M. Regulation of photosynthesis through source: sink imbalance in citrus is mediated by carbohydrate content in leaves. Physiologia Plantarum, v.116, p.563-572, 2002.

KRAMER, P.J.; BOYER, J.S. Water relations of plants and soils. San Diego: Academic, 1995. 495p.

LAMBERS, H.; CHAPIN III, F.S.; PONS, T.L. Plant physiological ecology. New York: Springer-Verlag, 1998. 604p.

LAWLOR, D.W.; CORNIC, G. Photosynthetic carbon and associated metabolism in relation to water deficits in higher plants. Plant, Cell and Environment, v.25, p.275-294, 2002.

LICHTENTHALER, H.K.; WELLBURN, A.R. Determinations of total carotenoids and chlorophylls $\mathrm{a}$ and $\mathrm{b}$ of leaf extracts in different solvents. Biochemical Society Transactions, v.11, p.591-592, 1983.

LÓPEZ-BUCIO, J.; HERNANDEZ-ABREU, E.; SÁNCHEZ-CALDERÓN, L.; NIETO JACOBO, M.F.; SIMPSON, J.; HERRERA-ESTRELLA, L. Phosphate availability alters architecture and causes changes in hormone sensitivity in the Arabidopsis root system. Plant Physiology, v.129, p.244-256, 2002.
MENARD, L.N.; MALAVOLTA, E. Estudos sobre alimentação mineral do cafeeiro. VII. Interação entre fósforo e ferro em cafeeiro (Coffea arabica L. var. Caturra KMC) cultivado em solução nutritiva. Anais da Escola Superior de Agricultura Luiz de Queiroz, v.19, p.23-33, 1962.

PINHEIRO, H.A.; DAMATTA, F.M.; CHAVES, A.R.M.; LOUREIRO, M.E.; DUCATTI, C. Drought tolerance is associated with rooting depth and stomatal control of water use in clones of Coffea canephora. Annals of Botany, v.96, p.101-108, 2005.

POZZA, A.A.A.; CARVALHO, J.G. de; GUIMARES, P.T.G.; FIGUEIREDO, F.C.; ARAÚJO, A.R. Suprimento do silicato de cálcio e a eficiência nutricional de variedades de cafeeiro. Revista Brasileira de Ciência do Solo, v.33, p.1705-1714, 2009.

RAIJ, B. van; ANDRADE, J.C. de; CANTARELLA, H.; QUAGGIO, J.A. (Ed.). Análise química para avaliação da fertilidade de solos tropicais. Campinas: IAC, 2001. 284p.

REIS JUNIOR, R. dos A.; MARTINEZ, H.E.P. Adição de Zn e absorção, translocação e utilização de $\mathrm{Zn}$ e $\mathrm{P}$ por cultivares de cafeeiro. Scientia Agricola, v.59, p.537-542, 2002.

RIBEIRO, R.V.; MACHADO, E.C.; SANTOS, M.G.; OLIVEIRA, R.F. Photosynthesis and water relations of well-watered orange plants as affected by winter and summer conditions. Photosynthetica, v.47, p.215-222, 2009.

ROHACEK, K. Chlorophyll fluorescence parameters: the definitions, photosynthetic meaning, and mutual relationships. Photosynthetica, v.40, p.13-29, 2002.

SANTOS, M.G. dos; RIBEIRO, R.V.; OLIVEIRA, R.F. de; MACHADO, E.C.; PIMENTEL, C. The role of inorganic phosphate on photosynthesis recovery of common bean after a mild water deficit. Plant Science, v.170, p.659-664, 2006.

SCHULTZ, H.R. Differences in hydraulic architecture account for near-isohydric and anisohydric behaviour of two field-grown Vitis vinifera L. cultivars during drought. Plant, Cell and Environment, v.26, p.1393-1405, 2003.

SHUBHRA; DAYAL, J.; GOSWAMI, C.L.; MUNJAL, R. Influence of phosphorus application on water relations, biochemical parameters and gum content in cluster bean under water deficit. Biologia Plantarum, v.48, p.445-448, 2004.

SILVA, V.A.; ANTUNES, W.C.; GUIMARÃES, B.L.S.; PAIVA, R.M.C.; SILVA, V. de F.; FERRÃO, M.A.G.; DAMATTA, F.M.; LOUREIRO, M.E. Resposta fisiológica de clone de café Conilon sensível à deficiência hídrica enxertado em porta-enxerto tolerante. Pesquisa Agropecuária Brasileira, v.45, p.457-464, 2010.

TORRES NETTO, A.; CAMPOSTRINI, E.; OLIVEIRA, J.G. de; BRESSAN-SMITH, R.E. Photosynthetic pigments, nitrogen, chlorophyll a fluorescence and SPAD-502 readings in coffee leaves. Scientia Horticulturae, v.104, p.199-209, 2005.

TOURNAIRE-ROUX, C.; SUTKA, M.; JAVOT, H.; GOUT, E.; GERBEAU, P.; LUU, D.-T.; BLIGNY, R.; MAUREL, C. Cytosolic $\mathrm{pH}$ regulates root water transport during anoxic stress through gating of aquaporins. Nature, v.425, p.393-397, 2003.

$\overline{\text { Recebido em } 29 \text { de junho de } 2010 \text { e aprovado em } 20 \text { de agosto de } 2010}$

Pesq. agropec. bras., Brasília, v.45, n.9, p.965-972, set. 2010 